University of Warwick institutional repository: http://go.warwick.ac.uk/wrap This paper is made available online in accordance with publisher policies. Please scroll down to view the document itself. Please refer to the repository record for this item and our policy information available from the repository home page for further information.

To see the final version of this paper please visit the publisher's website. Access to the published version may require a subscription.

Author(s): DAVID MOND and DUCO VAN STRATEN Article Title: MILNOR NUMBER EQUALS TJURINA NUMBER FOR FUNCTIONS ON SPACE CURVES

Year of publication: 2001 Link to published version: http://dx.doi.org/10.1112/S0024610700001320

Publisher statement: None 


\title{
MILNOR NUMBER EQUALS TJURINA NUMBER FOR FUNCTIONS ON SPACE CURVES
}

\author{
DAVID MOND AND DUCO VAN STRATEN
}

\begin{abstract}
The equality of the Milnor number and Tjurina number for functions on space curve singularities, as conjectured recently by V. Goryunov, is proved. As a consequence, the discriminant in such a situation is a free divisor.
\end{abstract}

\section{Introduction}

In many situations in complex geometry one can define a Milnor number that describes the rank of some vanishing homology, and a Tjurina number, the dimension of the base of a semi-universal deformation. For example, for a hypersurface singularity $X=V(f)$ one has

$$
\mu=\operatorname{dim} \mathcal{O} / J_{f}, \quad \tau=\operatorname{dim} \mathcal{O} /\left(f, J_{f}\right),
$$

so that $\mu \geqslant \tau$, with equality if $f$ is quasi-homogeneous, see [16]. Recently, $\mathrm{V}$. Goryunov [9] has studied functions on curve singularities $X \subset \mathbb{C}^{3}$, and conjectured, somewhat surprisingly, that the equality $\mu=\tau$ holds in such a situation, without any condition of quasi-homogeneity. For functions on smooth curves, the equality of $\mu$ and $\tau$ is of course so evident that it passes without notice: every non-constant germ in one variable is equivalent to $x^{n}$ for some $n>0$. We show in this note that Goryunov's conjecture is correct. In fact, the result follows from known results in homological algebra. Because many of these results are scattered over the literature, we try to be self-contained. We cannot, however, claim much originality.

\section{The Milnor number}

We will consider curve singularities $X$, by which we mean reduced, onedimensional germs $X \subset\left(\mathbb{C}^{N}, 0\right)$. We let $\mathcal{O}_{X}=\mathcal{O} / I$, where $\mathcal{O}:=\mathbb{C}\left\{x_{1}, x_{2}, \ldots, x_{N}\right\}$, be the local ring of $X$. Furthermore, we consider the dualising module

$$
\omega_{X}:=\operatorname{Ext}^{N-1}\left(\mathcal{O}_{X}, \Omega^{N}\right) .
$$

Here $\Omega^{N}$ is the module of $N$-forms on $\left(\mathbb{C}^{N}, 0\right)$. One can think of $\omega_{X}$, via the so-called class map cl: $\Omega_{X} \longrightarrow \omega_{X}$, as consisting of certain meromorphic differential forms on the curve. For an explanation, we refer to [10] and to Remark 2.4 and $\$ 4$ of this note.

A function $f \in \mathcal{O}_{X}$ can be considered as a map $f: X \longrightarrow S$, where $S$ is a smooth curve germ. We will always assume our functions $f$ to be non-constant on each branch, so that $\mathcal{O}_{X}$ becomes a finite and free $\mathcal{O}_{S}$-module via $f$.

Received 2 June 1999; revised 13 October 1999.

2000 Mathematics Subject Classification 32S20, 14B07 (primary), 14H20, 14H50 (secondary).

J. London Math. Soc. (2) 63 (2001) 177-187. (C) London Mathematical Society 2001. 
The ramification of the map $f: X \longrightarrow S$ can be measured as follows: the composition of $\mathcal{O}_{X} \stackrel{d f}{\longrightarrow} \Omega_{X}$ with the class map cl: $\Omega_{X} \longrightarrow \omega_{X}$ produces an injective map $\mathcal{O}_{X} \longrightarrow \omega_{X}$, whose cokernel we denote by $\mathscr{M}_{f}$ :

$$
0 \longrightarrow \mathcal{O}_{X} \longrightarrow \omega_{X} \longrightarrow \mathscr{M}_{f} \longrightarrow 0 .
$$

It is a module of finite length. We will denote by $\mathscr{R}_{f}$ the short exact sequence by which we define $\mathscr{M}_{f}$.

Definition 2.1. We define the Milnor number of $f: X \longrightarrow S$ as

$$
\mu(f):=\operatorname{dim} \mathscr{M}_{f} .
$$

Proposition 2.2. The Milnor number $\mu$ is conserved under simultaneous deformation of $f$ and $X$.

Proof. Consider a one-parameter deformation $\mathscr{X} \longrightarrow T$ of $X$ over $T$, and a lift of $f$ to a function $F: \mathscr{X} \longrightarrow S$. Because $X$ is assumed to be reduced, $\omega_{\mathscr{X}}$ is $T$-flat, and the snake lemma, applied to the injective morphism of complexes $\mathscr{R}_{F} \stackrel{t}{\longrightarrow} \mathscr{R}_{F}$ (with cokernel $\mathscr{R}_{f}$ ) gives the short exact sequence

$$
0 \longrightarrow \mathscr{M}_{F} \stackrel{{ }^{t}}{\longrightarrow} \mathscr{M}_{F} \longrightarrow M_{f} \longrightarrow 0 .
$$

Hence $\mathscr{M}_{F}$ is $\mathcal{O}_{T}$-flat, and therefore $\mathcal{O}_{T}$ free. It follows that $\mu$ is conserved in any 1parameter deformation of $X \stackrel{f}{\longrightarrow} S$, and thus in any deformation.

REMARK 2.3. If $\mathscr{X} \longrightarrow T$ is a smoothing of $X$, and $F$ is generic, then $\mu(f)$ counts the number of critical points of $f_{t}=F_{\mid X_{t}}$. These can be thought of as the points where the smooth curve $X_{t}$ is tangent to the level hypersurfaces of $F$. For at any such point, $\omega_{X_{t}}=\Omega_{X_{t}}^{1}$, and our module $\mathscr{M}_{f_{t}}$ is just $\Omega_{X_{t}}^{1} / d f_{t} \wedge \mathcal{O}_{X_{t}}$, and thus isomorphic to the usual jacobian algebra $\mathcal{O}_{X_{t}} / J_{f_{t}}$. Hence an ordinary tangency of $X_{t}$ to the level hypersurface of $F$ will contribute 1 to the total. In fact these are the only points at which length $\left(\mathscr{M}_{f_{t}}\right)$ is equal to 1 .

ReMark 2.4. (1) The module $\omega_{X}$ can be identified with $\operatorname{Hom}_{S}\left(\mathcal{O}_{X}, \omega_{S}\right)$ ([6, Theorem 3.3.7(b)]). After a choice of generator for $\omega_{S}, \omega_{S}=\mathcal{O}_{S}$, and so $\omega_{X} \simeq \operatorname{Hom}_{S}\left(\mathcal{O}_{X}, \mathcal{O}_{S}\right)$. Let $Q\left(\mathcal{O}_{X}\right)$ and $Q\left(\mathcal{O}_{S}\right)$ denote the total rings of fractions of $\mathcal{O}_{X}$ and $\mathcal{O}_{S}$ respectively, and let $\operatorname{tr}: \mathcal{O}_{X} \longrightarrow \mathcal{O}_{S}$ denote the trace map, which associates to each element $g$ of $\mathcal{O}_{X}$ the trace of the $Q\left(\mathcal{O}_{S}\right)$-linear endomorphism of $Q\left(\mathcal{O}_{X}\right)$ induced by multiplication by $g$. (The trace of a function $g \in \mathcal{O}_{X}$ can also be thought of as the function $\operatorname{tr}(g) \in \mathcal{O}_{S}$ whose value at a regular value $s \in S$ is the sum of the values of $g$ at the preimages of $s$.) As $\omega_{X}$ is maximal Cohen-Macaulay of rank 1, every element of $\operatorname{Hom}_{S}\left(\mathcal{O}_{X}, \mathcal{O}_{S}\right)$ is a $Q\left(\mathcal{O}_{X}\right)$-multiple of tr, that is,

$$
\operatorname{Hom}_{S}\left(\mathcal{O}_{X}, \mathcal{O}_{S}\right)=\left\{g \in Q\left(\mathcal{O}_{X}\right) \mid \operatorname{tr}(g h) \in \mathcal{O}_{S} \forall h \in \mathcal{O}_{X}\right\} \cdot \operatorname{tr} .
$$

The submodule of $Q\left(\mathcal{O}_{X}\right)$ defined here is the classical complementary module, usually denoted $\mathscr{L}_{X / S}$. Thus, we have shown that

$$
\omega_{X} \simeq \mathscr{L}_{X / S}
$$


Thought of as a module of meromorphic forms on $X, \omega_{X}=\mathscr{L}_{X / S} \cdot d f$, and the quotient $\mathscr{M}_{f}$ with which we defined the Milnor number $\mu$, is just $\left(\mathscr{L}_{X / S} \cdot d f\right) /\left(\mathcal{O}_{X} \cdot d f\right)$, or simply $\mathscr{L}_{X / S} / \mathcal{O}_{X}$.

(2) The module $\left(\mathscr{L}_{X / S} \cdot d f\right) /\left(\mathcal{O}_{X} \cdot d f\right)$ is also studied in [15], under the name $R^{+}(d f)$. It supports a natural quadratic form, induced by the residue pairing, and in [15] the signature of this form is used to compute the number of branches of the curve-germ $X$ and of its real part.

(3) There are many other ways to define numbers that are related to the ramification of the map $f: X \longrightarrow S$. In $\S 5$ we will see that if $X \subset \mathbb{C}^{3}$ is a space curve singularity, many of these numbers agree.

\section{The Tjurina number}

Given any map $f: X \longrightarrow S$ of analytic germs, there is a so-called (analytic)

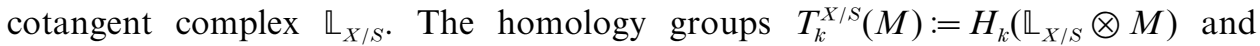
cohomology groups $T_{X / S}^{k}(M):=H^{k}\left(\operatorname{Hom}\left(\mathbb{Q}_{X / S}, M\right)\right)$ form a (co)-homology theory for $\mathcal{O}_{X}$-modules $M$. The modules $T_{k}^{X / S}:=T_{k}^{X / S}\left(\mathcal{O}_{X}\right)$ and $T_{X / S}^{k}:=T_{X / S}^{k}\left(\mathcal{O}_{X}\right)$ are important in the deformation theory of the map $f: X \longrightarrow S$.

$T_{0}^{X / S}$ is the module of relative Kähler 1-forms $\Omega_{X / S}, T_{X / S}^{0}$ is the module of relative vector fields $\Theta_{X / S}$ on $X$ over $S$. The other $T_{k}$ and $T^{k}$ are to be thought of as higher derived versions of these, and are supported inside the critical locus of the map. For a good overview, we refer to [3] and also [13]. Most remarkable is the module $T_{X / S}^{1}$, which has the interpretation as space of infinitesimal deformations of the map $f: X \longrightarrow S$, where we keep $S$ fixed, but are allowed to deform $X$ in any flat way, and the map in an arbitrary way. Isomorphisms are defined by obvious diagrams, always with identity on $S$. This leads to the following very general definition.

Definition 3.1. The Tjurina number of $f: X \longrightarrow S$ is

$$
\tau(f):=\operatorname{dim} T_{X / S}^{1} .
$$

Now let us return to the case of a function $f: X \longrightarrow S$ on a curve singularity. We remark that a space curve has a semi-universal deformation whose base is a smooth space of dimension $\tau=\operatorname{dim} T_{X / k}^{1}$ (see, for example, [17]). Similarly, standard arguments show that the base of a semi-universal deformation of the function $f: X \longrightarrow S$ is smooth of dimension $\tau(f)$.

ExAmple 3.2. For a Morse point, $\tau(f)=1$. Once again, this is the only case where the value of the invariant is 1 .

The modules $T_{k}$ and $T^{k}$ for $k \leqslant 2$ can be computed using the LichtenbaumSchlessinger complex. One starts with $X \subset\left(\mathbb{C}^{N}, 0\right)$ and extends the map $X \longrightarrow S$ to $\left(\mathbb{C}^{N}, 0\right) \longrightarrow S$. Choose generators of the ideal $\mathscr{J}$ of $X$, and let $\mathscr{R}$ denote the module of relations between the generators. That is, we have an exact sequence

$$
0 \longrightarrow \mathscr{R} \longrightarrow \mathcal{O}^{k} \longrightarrow \mathscr{J} \longrightarrow 0 .
$$

Let $\mathscr{R}_{0} \subset \mathscr{R}$ be the module of Koszul relations between the generators of $\mathscr{J}$. Consider the complex

$$
\mathbb{L}^{\leqslant 2}: \mathscr{R} / \mathscr{R}_{0} \longrightarrow \mathcal{O}^{k} \otimes \mathcal{O}_{X} \longrightarrow \Omega_{\mathbb{C}^{N} / S} \otimes \mathcal{O}_{X} .
$$


The homology groups of this complex are the $T_{k}^{X / S}$, the cohomology groups of its $\mathcal{O}_{X}$-dual the $T_{X / S}^{k}$, for $k=0,1,2$.

Proposition 3.3. Assume that $T_{X / S}^{2}=0$ and that $X$ is smoothable. Then

$$
\mu(f)=\tau(f) .
$$

Proof. Consider a one-parameter smoothing $\mathscr{X} \longrightarrow T$, and a function $F: \mathscr{X} \longrightarrow S$. Let $t$ be a local parameter on $T$. Put $\mathscr{S}=S \times T$; we have an obvious map $\mathscr{X} \longrightarrow \mathscr{S}$. The sequence

$$
0 \longrightarrow \mathcal{O}_{X} \stackrel{t}{\longrightarrow} \mathcal{O}_{X} \longrightarrow \mathcal{O}_{X} \longrightarrow 0
$$

induces a long exact sequence

$$
\ldots \longrightarrow T_{\mathscr{X} / \mathscr{S}}^{k} \longrightarrow T_{\mathscr{X} / \mathscr{S}}^{k} \longrightarrow T_{X / S}^{k} \longrightarrow T_{\mathscr{X} / \mathscr{S}}^{k+1} \longrightarrow \ldots
$$

However, $T_{X / S}^{0}=0$, as there are no relative vector fields for $f: X \longrightarrow S$, as the fibres are discrete. By assumption, $T_{X / S}^{2}=0$, so, since $T_{X, \mathscr{S}}^{2}$ is Artinian, the long exact sequence contains the short exact sequence

$$
0 \longrightarrow T_{\mathscr{X} / \mathscr{S}}^{1} \stackrel{{ }^{t}}{\longrightarrow} T_{\mathscr{X} / \mathscr{S}}^{1} \longrightarrow T_{X / S}^{1} \longrightarrow 0 .
$$

Thus $T_{X / \mathscr{S}}^{1}$ is $T$-flat, and by the same argument as in the proof of Proposition 2.2, $\tau$ is conserved in the family. At each point on the smooth curve $X_{t}, \tau\left(f_{t}\right)$ and $\mu\left(f_{t}\right)$ are equal, and by the conservation of each number in the deformation, the result follows.

The reader will note that we do not exhibit an explicit isomorphism $\mathscr{M}_{f} \longrightarrow T_{X / S}^{1}$.

REMARK 3.4. (1) Associated to the composite of ring homomorphisms

$$
\mathbb{C} \longrightarrow \mathcal{O}_{S} \longrightarrow \mathcal{O}_{X}
$$

there is a long exact sequence of cotangent cohomology, the Zariski-Jacobi sequence, which runs

$$
\ldots \longrightarrow T_{X / S}^{i} \longrightarrow T_{X / \mathbb{C}}^{i} \longrightarrow T_{S / C}^{i} \longrightarrow T_{X / S}^{i+1} \longrightarrow \ldots
$$

Here $T_{X / \mathbb{C}}^{i}$ and $T_{S / \mathbb{C}}^{i}$ are just $T_{X}^{i}$ and $T_{S}^{i}$ respectively; since $S$ is smooth, $T_{S}^{i}=0$ for $i \geqslant 1$ (and recall that $T_{S}^{0}=\theta_{S}$ ), and it follows that $T_{X / S}^{i}=T_{X}^{i}$ for $i \geqslant 2$. So the condition in the theorem is equivalent to $T_{X}^{2}=0$.

(2) It is known that $T_{X}^{2}=0$ for space curves and Gorenstein curves in $\mathbb{C}^{4}$, so in these cases Proposition 3.3 holds $[\mathbf{1 2}, \mathbf{1 9}]$. See also $\S 4$ and in particular Corollary 4.3.

(3) In fact, the proposition holds more generally for all unobstructed curves (that is, curves in which the obstruction map $T_{X / S}^{1} \longrightarrow T_{X / S}^{2}$ is equal to 0 . For a general smoothable curve one can use a variant of [11] to see that the image of $T_{X / \mathscr{S}}^{1} \longrightarrow T_{X / S}^{1}$ has a dimension that is equal to the dimension of the component of the deformation space on which the smoothing occurs. In particular, this dimension is equal to $\mu(f)$, and thus all components have the same, computable dimension.

(4) In the same way one may prove that the relative $T^{1}$ is Cohen-Macaulay over the semi-universal deformation of an unobstructed curve. From this fact one deduces, as in [18], that the discriminant is a free divisor.

(5) In general, one can use the long exact sequences for the relative $T^{k}$ to find inequalities between $\mu$ and partial Euler characteristics of the $T^{i}$; see [13]. 


\section{The first Koszul homology}

We will now take a closer look at space curves $X \subset \mathbb{C}^{3}$. The salient feature of this case is that one still has complete control over the structure of the equations of $X$, which are obtained as the $n \times n$ minors of some $(n+1) \times n$-matrix $M$. That is, writing $\mathcal{O}$ for the ring of functions on $\mathbb{C}^{3}, \mathcal{O}_{X}$ has a free $\mathcal{O}$-resolution of the form

$$
0 \longrightarrow \mathcal{O}^{n} \stackrel{M}{\longrightarrow} \mathcal{O}^{n+1} \stackrel{\Delta}{\longrightarrow} \mathcal{O} \longrightarrow \mathcal{O}_{X} \longrightarrow 0 .
$$

Here $\Delta$ is the map with components $\Delta_{i}$, which is the minor of $M$ obtained by deleting the $i$ th row.

Note that $\mathscr{O}^{n}$ is the module $\mathscr{R}$ of relations between the $\Delta_{i}$. The module $\mathscr{R}_{0}$ is generated by the Koszul (or trivial) relations $\Delta_{i} e_{j}-\Delta_{j} e_{i}$. To describe these elements inside $\mathcal{O}^{n}$, we use the map

$$
\Gamma: \mathcal{O}_{2}^{\left(\begin{array}{c}
n+1 \\
2
\end{array}\right)}=\wedge^{2} \mathcal{O}^{n+1} \longrightarrow \mathcal{O}^{n}
$$

whose entries consist of the $(n-1) \times(n-1)$ minors of $M$ :

$$
\Gamma\left(e_{i} \wedge e_{j}\right)=\sum_{k=1}^{n} \operatorname{det} \Gamma_{i, j}^{k} e_{k},
$$

where $\Gamma_{i, j}^{k}$ is the matrix obtained from $M$ by deleting $i$ th and $j$ th rows and $k$ th column. The composition

$$
\wedge^{2} \mathcal{O}^{n+1} \stackrel{\Gamma}{\longrightarrow} \mathcal{O}^{n} \stackrel{M}{\longrightarrow} \mathcal{O}^{n+1}
$$

maps $e_{i} \wedge e_{j}$ to the Koszul relation $\Delta_{i} e_{j}-\Delta_{j} e_{i}$.

The cokernel of the map $\Gamma$ is the first Koszul homology module of the $\Delta_{i}$ :

$$
\mathscr{R} / \mathscr{R}_{0}=H_{1}(\mathcal{O}, \underline{\Delta})=: H_{1} .
$$

It is a fundamental result that $H_{1}$ is a torsion free $\mathcal{O}_{X}$-module, and this fact has some useful consequences. Let us see why this is the case.

As we are going to work with various minors, it is useful to describe the above resolution in a basis-free manner and work in complete generality. Let $R$ be for the moment any ring, and let $\mathscr{F}$ and $\mathscr{G}$ be free $R$-modules of rank $n$ and $(n+1)$ respectively. We fix isomorphisms

$$
\wedge^{n} \mathscr{F} \cong R, \quad \wedge^{n+1} \mathscr{G} \cong R .
$$

Note that with these fixed identifications, one has

$$
\wedge^{n-p} \mathscr{F}=\wedge^{p} \mathscr{F}^{*}, \quad \wedge^{n+1-p \mathscr{G}}=\wedge^{p} \mathscr{G}^{*} .
$$

For any $R$-module $N$ we denote its dual by $N^{*}:=\operatorname{Hom}_{R}(N, R)$. Given any homomorphism $M: \mathscr{F} \longrightarrow \mathscr{G}$, one derives from it other maps like

$$
\wedge^{p} M: \wedge^{p} \mathscr{F} \longrightarrow \wedge^{p} \mathscr{G}, M^{*}: \mathscr{G}^{*} \longrightarrow \mathscr{F}^{*}, \wedge^{p}\left(M^{*}\right): \wedge^{p} \mathscr{G}^{*} \longrightarrow \wedge^{p} \mathscr{F}^{*},
$$

and so on. In particular, we get composable maps

$$
\wedge^{p} \mathscr{F} \longrightarrow \wedge^{p} \mathscr{G}=\wedge^{n+1-p \mathscr{G} *} \longrightarrow \wedge^{n+1-p} \mathscr{\mathscr { F }} *=\wedge^{p-1} \mathscr{F} .
$$

It follows from the Laplace expansion rule of determinants that the composition of these two maps is zero, so that we get complexes. (We put the right-most module at spot zero, so that the complex lives in negative degrees.) 
For $p=1$, we get the complex $0 \longrightarrow \mathscr{F} \longrightarrow \mathscr{G} \longrightarrow R \longrightarrow 0$; for $p=n$ we get its dual $0 \longrightarrow R \longrightarrow \mathscr{G}^{*} \longrightarrow \mathscr{F}^{*} \longrightarrow 0$. We are most interested in the case $p=2$ :

$$
0 \longrightarrow \wedge^{2} \mathscr{F} \longrightarrow \wedge^{2} \mathscr{G} \longrightarrow \mathscr{F} \longrightarrow 0 \text {. }
$$

These complexes were first considered in [7] and [2].

Proposition 4.1 (cf. [2, Lemma 1.6]). Let $I \subset R$ be the ideal generated by the maximal minors of $M$. Assume that the local cohomologies $H_{I}^{i}(R)=0$ for $i=0,1$ (that is, depth $(R) \geqslant 2)$. Then the above complexes are acyclic.

Proof. This is essentially the Lemme d'Acyclicité (see also [15, A.2, A.3]).

For any $R$-module $M$, let

$$
C^{p}(M)=\sum_{1 \leqslant i_{1}<\ldots<i_{p} \leqslant n+1} M_{f_{i_{1}}, \ldots, f_{i_{p}}}
$$

be the $p$ th Cech module of $M$, with respect to a set of generators $f_{i}$ of $I$, so that $C^{\bullet}(M)$ computes the local cohomology $H_{I}^{\bullet}(M)$ of $M$. Let $F_{\bullet}$ denote any of the complexes whose acyclicity we wish to prove, and consider the double complex $C^{\bullet}\left(F_{\bullet}\right)$. Since the cohomology of $F_{\bullet}$ is supported on $\operatorname{Spec}(R / I)$, it follows that for any $f \in I$, the complex $\left(F_{\bullet}\right)_{(f)}$ is acyclic, and thus the same is true for all of the complexes $C^{p}\left(F_{\bullet}\right)$. So the total complex computes the cohomology of $F_{\bullet}$. On the other hand, the assumption of vanishing of local cohomology tells us that if we first take vertical cohomology, we get an $E_{1}$ term looking like

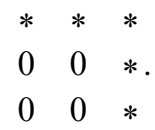

Because the associated spectral sequence also computes the cohomology of the total complex, the proposition follows immediately.

Corollary 4.2. (1) If $R$ is Cohen-Macaulay, and I an ideal of codimension $\geqslant 2$, then it follows that the codimension equals 2.

(2) Under the above assumptions, we have exact sequences

$$
\begin{gathered}
0 \longrightarrow \mathscr{F} \longrightarrow \mathscr{G} \longrightarrow R \longrightarrow R / I \longrightarrow 0 \\
0 \longrightarrow \wedge^{2} \mathscr{F} \longrightarrow \wedge^{2} \mathscr{G} \longrightarrow F \longrightarrow H_{1} \longrightarrow 0 .
\end{gathered}
$$

In particular $R / I$ and $H_{1}$ are Cohen-Macaulay.

Hence, for a space curve, the module $H_{1}=\mathscr{R} / \mathscr{R}_{0}$ is torsion free.

Corollary 4.3. If $X$ is reduced Cohen-Macaulay of codimension 2, then $T_{2}^{X}=0$.

Proof. From the Lichtenbaum-Schlessinger complex, $T_{2}^{X}=\operatorname{ker}\left(H_{1} \longrightarrow \ldots\right)$. However, as $T_{2}$ is concentrated on the singular locus of $X$ and $H_{1}$ is Cohen-Macaulay, it follows that $T_{2}$ must be zero.

Remark 4.4. (1) The other complexes resolve the higher Koszul homologies $H_{i}$. Therefore all of these are Cohen-Macaulay too. 
(2) It was shown by Huneke [14] that the Cohen-Macaulay property of all Koszul homologies $H_{i}$ is preserved under linkage. In particular, these results holds if $X$ is linked to a complete intersection. This in particular applies to space curves: use induction and the fact that a curve defined by the minors of an $(n+1) \times n$ matrix is linked via the curve defined by two of these minors to the curve defined by the minors of their common $(n-1) \times n$ submatrix.

Another feature of space curves is the close relation between the $\mathcal{O}_{X^{-}}$dual $\omega^{*}$ of the dualising module $\omega_{X}$, and $H_{1}$, which implies that $\omega_{X}^{*}$ behaves well under flat deformation of $X$, and gives the vanishing of $T_{X}^{2}$.

Start with the resolution of $\omega_{X}$ :

$$
0 \longrightarrow R \longrightarrow \mathscr{G}^{*} \longrightarrow \mathscr{F}^{*} \longrightarrow \omega_{X} \longrightarrow 0 .
$$

Applying $\operatorname{Hom}_{X}\left(-, \mathcal{O}_{X}\right)$ to this, we get $\omega_{X}^{*}$ as kernel:

$$
0 \longrightarrow \omega_{X}^{*} \longrightarrow \mathscr{F} \otimes \mathcal{O}_{X} \longrightarrow \mathscr{G} \otimes \mathcal{O}_{X} .
$$

On the other hand, the composition

$$
\wedge^{2} \mathscr{G} \longrightarrow \mathscr{F} \longrightarrow \mathscr{G}
$$

is the zero-map after tensoring with $\mathcal{O}_{X}$; hence the first map factors to $\omega_{X}^{*}$. We let $\mathscr{C}$ be the cokernel as follows.

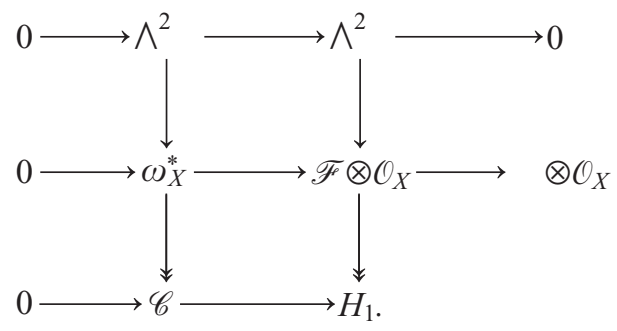

A local calculation shows that $\mathscr{C}$ is supported at the singular point; from the snake lemma $\mathscr{C} \longrightarrow H_{1}$ is injective. As $H_{1}$ is torsion free, we get $\mathscr{C}=0$.

Hence

and

$$
\wedge^{2} \mathscr{G} \longrightarrow \omega_{X}^{*} \longrightarrow 0
$$

$$
0 \longrightarrow \omega_{X}^{*} \longrightarrow \mathscr{F} \otimes \mathcal{O}_{X} \longrightarrow H_{1} \longrightarrow 0
$$

are exact sequences.

Corollary 4.5. If $X$ is reduced Cohen-Macaulay of codimension 2, then $T_{X}^{2}=0$.

Proof. Dualising the exact sequence

$$
0 \longrightarrow \omega_{X}^{*} \longrightarrow \mathscr{F} \otimes \mathcal{O}_{X} \longrightarrow H_{1} \longrightarrow 0,
$$

we get the exact sequence

$$
0 \longrightarrow H_{1}^{*} \longrightarrow \mathscr{F}^{*} \otimes \mathcal{O}_{X} \longrightarrow \omega_{X}^{* *} \longrightarrow \operatorname{Ext}^{1}\left(H_{1}, \mathcal{O}_{X}\right) \longrightarrow 0 .
$$

However, $\mathscr{F}^{*} \otimes \mathcal{O}_{X}$ has just $\omega_{X}$ as image in $\omega_{X}^{* *}$; hence we have an exact sequence

$$
0 \longrightarrow H_{1}^{*} \longrightarrow \mathscr{F}^{*} \otimes \mathcal{O}_{X} \longrightarrow \omega_{X} \longrightarrow 0 \text {. }
$$


On the other hand, tensoring the resolution of $\omega_{X}$ with $\mathcal{O}_{X}$ gives an exact sequence

$$
\mathscr{C}^{*} \otimes \mathcal{O}_{X} \longrightarrow \mathscr{F}^{*} \otimes \mathcal{O}_{X} \longrightarrow \omega_{X} \longrightarrow 0
$$

from which we conclude that we have a surjection

$$
\mathscr{G}^{*} \otimes \mathcal{O}_{X} \longrightarrow H_{1}^{*} \text {. }
$$

The cokernel of this map, however, is by definition $T_{X}^{2}$.

\section{Kähler and Dedekind different}

In $\S 2$ we defined the Milnor number of a function on an arbitrary curve as the length of the cokernel $\mathcal{O}_{X} \longrightarrow \omega_{X}$. This cokernel has the advantage of being flat under any deformation, but has the disadvantage that in general it may have many generators. It would be computationally better to have a description of colength of some Jacobian-type ideal $J \subset \mathcal{O}_{X}$. The first idea that comes to mind is to use the module $\Theta_{X}$ of all vector fields on $X$, and evaluate them on $f$ to get $\Theta_{X}(f) \subset \mathcal{O}_{X}$. In this way we get the Bruce-Roberts number [5]:

$$
\mu_{B R}(f):=\operatorname{dim} \mathcal{O}_{X} / \Theta_{X}(f) .
$$

As vector fields do not lift automatically over deformations, this will behave badly non-flat, and thus cannot possibly be equal to the topological number $\mu(f)$.

A better idea is to look at the transpose of the map $\mathcal{O}_{X} \longrightarrow \omega_{X}$. It gives a map $\omega_{X}^{*} \longrightarrow \mathcal{O}_{X}$. Via the transpose of the class map, $\omega^{*}$ is mapped injectively into $\Theta_{X}$; we denote its image by $\theta_{X}^{s}$. The image of $\omega_{X}^{*}$ in $\mathcal{O}_{X}$ is the ideal obtained by evaluating these special vector fields (the elements of $\theta_{X}^{s}$ ) on $f$. The ideal

$$
\mathscr{D}_{D}(X / S):=\omega_{X}^{*}(f) \subset \mathcal{O}_{X}
$$

is classically known as the Dedekind different of the map $f: X \longrightarrow S$; it is the inverse ideal of the complementary module $\mathscr{L}_{X / S}$.

DEFINITION 5.1.

$$
\mu_{D}(f):=\operatorname{dim} \mathcal{O}_{X} / \mathscr{D}_{D}(X / S) .
$$

Proposition 5.2. Let $f: X \longrightarrow S$ be a function on a space curve singularity. Then

$$
\mu(f)=\mu_{D}(f) .
$$

Proof. It follows from $\S 4$ that $\omega_{X}^{*}$ behaves well under deformation. Let $\mathscr{X} \longrightarrow T$ be a flat deformation of $X$; applying $\operatorname{Hom}_{\mathcal{O}_{x}}\left(, \mathcal{O}_{\mathscr{X}}\right)$ to the exact sequence

$$
0 \longrightarrow \omega_{x} \stackrel{t \cdot}{\longrightarrow} \omega_{x} \longrightarrow \omega_{X} \longrightarrow 0
$$

we get the sequence

$$
0 \longrightarrow \omega_{X}^{*} \stackrel{t \cdot}{\longrightarrow} \omega_{X}^{*} \longrightarrow \omega_{X}^{*} \longrightarrow 0,
$$

which we claim is also exact. The only point in doubt is the zero at the right-hand side; this comes from the fact that we have a priori generators for $\omega_{X}^{*}: \wedge^{2} \mathscr{G} \longrightarrow \omega_{X}^{*}$. See also [18], where the result is deduced in a more complicated manner from $T_{2}^{X}=0$. 
By dualising the relative class map $\mathrm{cl}_{X / T}: \Omega_{\mathscr{X} / T}^{1} \longrightarrow \omega_{\mathscr{X}}$ we get an inclusion $\omega_{\mathscr{X}}^{*} \longleftrightarrow \theta_{\mathscr{X} / T}$; denoting its image by $\theta_{\mathscr{X} / T}^{s}$, the exact sequence of $\omega^{*}$ 's becomes

$$
0 \longrightarrow \theta_{\mathscr{X} / T}^{s} \stackrel{t}{\longrightarrow} \theta_{x / T}^{s} \longrightarrow \theta_{X}^{s} \longrightarrow 0 .
$$

Now evaluate the special vector fields on a lifting $F$ of $f$. Note that a function which is not constant on any branch of a curve cannot annihilate any non-torsion vector field, and that the modules of special vector fields are torsion-free. We therefore get an exact sequence

$$
0 \longrightarrow \mathcal{O}_{X} /\left(\theta_{X / T}^{s} \cdot F\right) \stackrel{t}{\longrightarrow} \mathcal{O}_{X} /\left(\theta_{X / T}^{s} \cdot F\right) \longrightarrow \mathcal{O}_{X} / \mathscr{D}_{D}(X / S) \longrightarrow 0,
$$

from which the proposition follows.

As we know the generators of $\omega_{X}^{*}$, we can be more explicit about the ideal $\mathscr{D}_{D}(X / S)$.

Proposition 5.3. The element $e_{i} \wedge e_{j}$ of $\wedge^{2} \mathscr{G}$ maps to the vector field

in $\omega_{X}^{*} \subset \Theta_{X}$.

$$
\operatorname{det}\left(\begin{array}{ccc}
\frac{\partial}{\partial x} & \frac{\partial}{\partial y} & \frac{\partial}{\partial z} \\
\frac{\partial \Delta_{i}}{\partial x} & \frac{\partial \Delta_{i}}{\partial y} & \frac{\partial \Delta_{i}}{\partial z} \\
\frac{\partial \Delta_{j}}{\partial x} & \frac{\partial \Delta_{j}}{\partial y} & \frac{\partial \Delta_{j}}{\partial z}
\end{array}\right)
$$

Proof. According to [1], the class map $\Omega_{X} \longrightarrow \omega_{X}=\operatorname{Ext}_{\mathscr{Q}}^{2}\left(\mathcal{O}_{X}, \Omega^{3}\right)$ is the following: let $\alpha \in \Omega^{1}$ be a lift of a 1 -form from $\Omega_{X}$. The element

$$
\frac{1}{2} \alpha \wedge d \Delta \wedge d M
$$

can be considered as an element of $\mathscr{F} * \otimes \Omega^{3}$, which represents the element $\operatorname{cl}(\alpha)$ in $\operatorname{Ext}^{2}\left(\mathcal{O}_{X}, \Omega^{3}\right)$. In order to simplify notation, we will fix a volume form $d V=d x \wedge d y \wedge d z$ and freely divide and multiply by it, when necessary. An element $\phi \in \omega_{X}^{*}$ is a special element of $\mathscr{F} \otimes \mathcal{O}_{X}$; considered as vector field (that is, in $\theta_{X}^{s}$ ) it is

$$
\phi(\operatorname{cl}(d x)) \frac{\partial}{\partial x}+\phi(\operatorname{cl}(d y)) \frac{\partial}{\partial y}+\phi(\operatorname{cl}(d z)) \frac{\partial}{\partial z} .
$$

Using a basis $e_{i}$ for $\mathscr{G}$ and $f_{j}$ for $\mathscr{F}$, the image of the element $e_{i} \wedge e_{j}$ under $\Gamma: \wedge^{2} \mathscr{G} \longrightarrow \mathscr{F}$ is $\Gamma_{i, j}^{k} f_{k}$, where $\Gamma_{i, j}^{k}$ is the minor obtained from $M$ by deleting the $i$ th and $j$ th rows and $k$ th column. These elements generate $\omega_{X}^{*}$. For these elements one has:

$$
\Gamma\left(e_{i} \wedge e_{j}\right)(\operatorname{cl}(\alpha))=\frac{1}{2} \alpha \wedge d \Delta_{p} \wedge d\left(M_{k}^{p}\right) \Gamma_{i, j}^{k}
$$

where we use the Einstein summation convention. Because $\Delta_{p} M_{k}^{p}=0$ we have $d \Delta_{p} M_{k}^{p}+\Delta_{p} d M_{k}^{p}=0$, so, modulo the ideal generated by the $\Delta_{i}$, we have

$$
d \Delta_{p} \wedge d\left(M_{k}^{p} \Gamma_{i, j}^{k}\right)=d \Delta_{p} \wedge d\left(M_{k}^{p}\right) \Gamma_{i, j}^{k} .
$$


Now we had $M_{k}^{p} \Gamma_{i, j}^{k}=\Delta_{i} \delta_{j}^{p}-\Delta_{j} \delta_{i}^{p}$; hence

That is, one has

$$
d \Delta_{p} \wedge d\left(M_{k}^{p} \Gamma_{i, j}^{k}\right)=2 d \Delta_{j} \wedge d \Delta_{i} .
$$

$$
\Gamma\left(e_{i} \wedge e_{j}\right)(\operatorname{cl}(\alpha))=\alpha \wedge d \Delta_{j} \wedge d \Delta_{i} .
$$

The formula follows (up to a sign).

The most obvious way to measure ramification is to look at the module $\Omega_{X / S}$ of relative Kähler differentials. The problem with it is that it does not have flatness properties needed to have a topological meaning. Therefore, instead, we look at the Fitting ideal $F_{0}\left(\Omega_{X / S}\right) \subset \mathcal{O}_{X}$, generated by the maximal minors of a presentation matrix for $\Omega_{X / S}$. It is also known under the name of Kähler different: $\mathscr{D}_{K}(X / S)=F_{0}\left(\Omega_{X / S}\right)$. We define

$$
\mu_{K}(f):=\operatorname{dim} \mathcal{O}_{X} / \mathscr{D}_{K}(X / S) .
$$

In general, one has an inclusion

$$
\mathscr{D}_{K}(X / S) \subset \mathscr{D}_{D}(X / S)
$$

and hence an inequality $\mu_{D}(f) \leqslant \mu_{K}(f)$, see $[\mathbf{4}, \mathbf{8}]$.

For space curves, one has equality as follows.

Proposition 5.4. If $X \subset\left(\mathbb{C}^{3}, 0\right)$ is a space curve singularity, then

hence

$$
\mathscr{D}_{K}(X / S)=\mathscr{D}_{D}(X / S) \text {; }
$$

$$
\mu(f)=\mu_{D}(f)=\mu_{K}(f) .
$$

Proof. The sequence

$$
\mathcal{O}_{X}^{n+2} \stackrel{J}{\longrightarrow} \mathcal{O}_{X}^{3}=\Omega_{\mathbb{C}^{3}} \otimes \mathcal{O}_{X} \longrightarrow \Omega_{X / S}
$$

is a presentation of $\Omega_{X / S}$. Here the matrix $J$ is

$$
\left(\begin{array}{cccc}
\frac{\partial f}{\partial x} & \frac{\partial \Delta_{1}}{\partial x} & \ldots & \frac{\partial \Delta_{n+1}}{\partial x} \\
\frac{\partial f}{\partial y} & \frac{\partial \Delta_{1}}{\partial y} & \ldots & \frac{\partial \Delta_{n+1}}{\partial y} \\
\frac{\partial f}{\partial z} & \frac{\partial \Delta_{1}}{\partial z} & \ldots & \frac{\partial \Delta_{n+1}}{\partial z}
\end{array}\right) .
$$

Thus, in view of the above inequality, the ideal of $3 \times 3$ minors $F_{0}\left(\Omega_{X / S}\right)$ is precisely $\mathscr{D}_{D}(X / S)$.

REMARK 5.5. One has

$$
\operatorname{dim} \Omega_{X / S}-\operatorname{dim} T_{1}^{X / S}=\mu(f)
$$

as one proves using $T_{2}=0$ and the argument of $\S 2$. Note that

$$
T_{1}^{X / S}=\operatorname{torsion}\left(I / I^{2}\right)=\int I / I^{2}
$$

is the module of non-trivial hypersurfaces singular along $X$. For a complete intersection it is zero. 


\section{References}

1. B. AngÉNiol and M. Lejeune-Jalabert, Calcul differentiel et classes characteristique en geometrie algébrique, Travaux en Cours 38 (Hermann, Paris, 1989).

2. L. Avramov and J. Herzog, 'The Koszul algebra of a codimension two embedding', Math. Z. 175 (1980) 249-260.

3. K. Behnke and J. A. Christophersen, 'Hypersurface sections and obstructions (rational surface singularities), Compositio Math. 77 (1991) 233-268.

4. R. BERGER, Über verschiedene Differentenbegriffe, Berichte Heidelberger Akademie der Wissenschaften I. Abhandlungen (1960).

5. J. W. BRUCE and R. M. Roberts, 'Critical points of functions on analytic varieties', Topology 27 (1988) 57-90.

6. W. Bruns and J. Herzog, Cohen-Macaulay rings, Cambridge Studies in Advanced Mathematics 39 (Cambridge University Press, 1993).

7. D. A. Buchsbaum and D. S. Rim, 'A generalised Koszul complex II', Trans. AMS 111 (1964) $197-224$.

8. R. BuchweITZ, On Zariski's criterion for equisingularity and non-smoothable monomial curves, thesis, Université Paris VII, 1981.

9. V. Goryunov, 'Functions on space curves', J. London Math. Soc. (2) 61 (2000) 807-822.

10. G.-M. Greuel and R.-O. Buchweitz, 'The Milnor number and deformations of complex curve singularities', Invent. Math. 58 (1980) 241-281.

11. G.-M. Greuel and E. Looljenga, 'The dimension of smoothing components', Duke Math. J. 52 (1985) 263-272.

12. J. Herzog, 'Deformationen von Cohen-Macaulay Algebren', J. Reine Angew. Math. 318 (1980) $83-105$.

13. J. Herzog and R. WALdi, 'Cotangent functors of curve singularities', Manuscripta Math. 55 (1986) $307-341$.

14. C. Huneke, 'Linkage and the Koszul homology of ideals', Amer. J. Math. 104 (1982) 1043-1062.

15. J. Montaldi and D. van Straten, 'One-forms on singular curves and the topology of real curve singularities', Topology 29 (1990) 501-510.

16. K. SAIto, 'Quasihomogene isolierte Singularitäten von Hyperflächen', Inv. Math. 14 (1971) 123-142.

17. M. Schaps, 'Deformations of Cohen-Macaulay schemes of codimension 2 and non-singular deformations of space-curves', Amer. J. Math. 99 (1977) 660-684.

18. D. van Straten, 'A note on the discriminant of a space curve', Manuscripta Math. 87 (1995) 167-177.

19. R. WALDI, 'Deformationen von Gorenstein Singularitäten der Kodimension', Math. Ann. 242 (1979) 201-208.

\section{University of Warwick Coventry CV4 $7 A L$}

mond@maths.warwick.ac.uk

\author{
Fachbereich Mathematik (17) \\ Johannes Gutenberg-Universität \\ D-55099 Mainz \\ Germany
}

straten@mathematik.uni-mainz.de 